\title{
Phagocytes whet their appetite
}

The engulfment ability of a phagocyte is controlled by its mitochondrial membrane potential, according to a study by Ravichandran and colleagues.

The authors reasoned that the 'ingestion' of metabolites from the cells that phagocytes engulf may affect their mitochondrial function. Indeed, phagocytes that had engulfed target cells had a higher mitochondrial membrane potential than non-engulfing phagocytes. This change was not observed when the phagocytes engulfed synthetic targets, indicating it may be the result of ingesting particular metabolites.

The phagocyte mitochondrial membrane potential returned to normal after a few hours, with no changes in ATP generation. On the basis of this, the authors speculated that uncoupling proteins (UCPs) may be involved, as they regulate mitochondrial membrane potential without generating ATP. Consistent

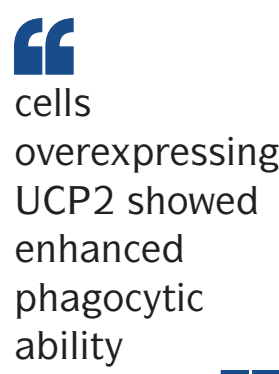

gु with this, UCP2 levels increased following phagocytosis of cellular, but not synthetic, targets, and UCP2-depleted cells were less efficient in phagocytosis.

Interestingly, cells overexpressing UCP2 showed enhanced phagocytic ability, could continue engulfing apoptotic cells for longer and had lower mitochondrial membrane potential than control cells. This indicates that UCP2 enhances the engulfment ability of phagocytes by decreasing their mitochondrial membrane potential. In agreement with this, phagocytes treated with compounds that artificially lower mitochondrial membrane potential showed enhanced phagocytic ability, whereas drugs that raise mitochondrial membrane potential inhibited phagocytosis.

Next, the authors asked whether UCP2 might somehow be sensing events that occur at the cell membrane. Indeed, they identified crosstalk between UCP2 and TIM4 (T cell immunoglobulin- and mucin domain-containing molecule 4), an engulfment receptor that binds phosphatidylserine on apoptotic cells.
Finally, the authors confirmed their findings in vivo. Bone marrow-derived macrophages from UCP2-deficient mice had higher mitochondrial membrane potential than control cells and showed reduced phagocytic ability ex vivo. Moreover, UCP2-deficient mice injected with dexamethasone (which triggers apoptosis in thymocytes) had higher total thymic numbers than control mice, and did not show a reduction in thymic size, both of which were caused by decreased apoptotic cell clearance.

This study reveals an unexpected link between low mitochondrial membrane potential, mediated by UCP2, and engulfment ability. Further studies will be required to determine how membrane potential affects phagocytosis and how UCP2 itself is activated.

Rachel David, Assistant Editor, Nature Reviews Molecular Cell Biology

ORIGINAL RESEARCH PAPER Park, D. et al.

Continued clearance of apoptotic cells critically depends on the phagocyte Ucp2 protein. Nature 21 Aug 2011 (doi: 10.1038/nature10340)

This article originally appeared in Nature Rev. Mol. Cell Biol. (doi:10.1038/nrm3192). 